\title{
Supervised ensemble learning methods towards automatically filtering Urdu fake news within social media
}

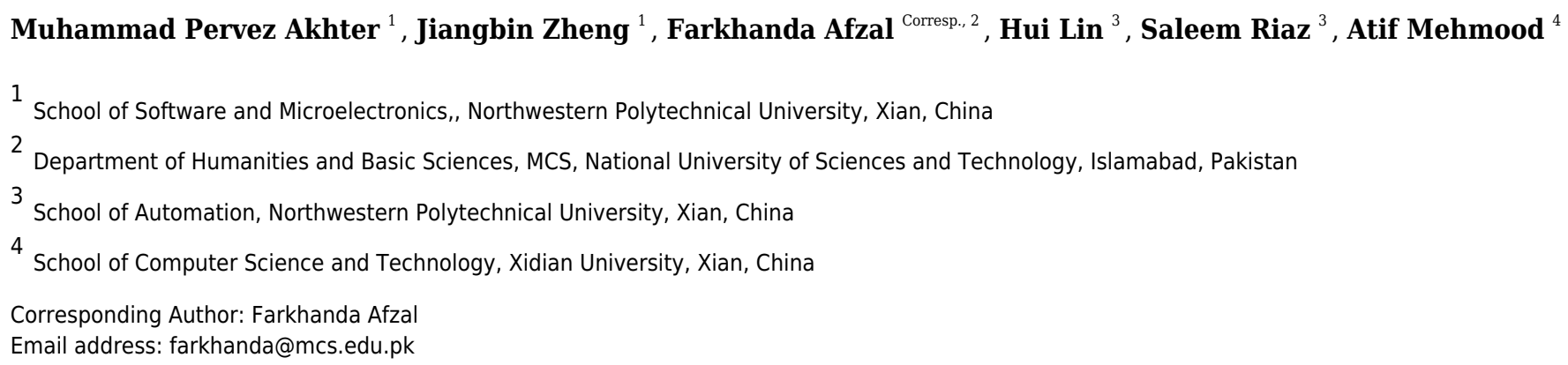

The popularity of the internet, smartphones, and social networks has contributed to the proliferation of misleading information like fake news and fake reviews on news blogs, online newspapers, and e-commerce applications. Fake news has a worldwide impact and potential to change political scenarios, deceive people into increasing product sales, defaming politicians or celebrities, and misguiding visitors to stop visiting a place or country. Therefore, it is vital to find automatic methods to detect fake news online. In several past studies, the focus was the English language, but the resource-poor languages have been completely ignored because of the scarcity of labeled corpus. In this study, we investigate this issue in the Urdu language. Our contribution is threefold. First, we design an annotated corpus of Urdu news articles for the fake news detection tasks. Second, we explore three individual machine learning models to detect fake news. Third, we use five ensemble learning methods to ensemble the base-predictors' predictions to improve the fake news detection system's overall performance. Our experiment results on two Urdu news corpora show the superiority of ensemble models over individual machine learning models. Three performance metrics balanced accuracy, the area under the curve, and mean absolute error used to find that Ensemble Selection and Vote models outperform the other machine learning and ensemble learning models. 


\section{Supervised ensemble learning methods towards} 2 automatically filtering Urdu fake news within social 3 media

Muhammad Pervez Akhter ${ }^{1}$, Zheng Jiangbin ${ }^{1}$, Farkhanda Afzal ${ }^{2}$, Lin Hui ${ }^{3}$, Saleem Riaz ${ }^{3}$, Atif Mehmood $^{4}$

7

${ }^{1}$ School of Software and Microelectronics, Northwestern Polytechnical University 710072 , Xian, P.R. China

2 Department of Humanities and Basic Sciences, MCS, National University of Sciences and Technology, Islamabad, Pakistan.

MCS, NUST, Islamabad, Pakistan.

\section{Abstract}

The popularity of the internet, smartphones, and social networks has contributed to the proliferation of misleading information like fake news and fake reviews on news blogs, online newspapers, and e-commerce applications. Fake news has a worldwide impact and potential to change political scenarios, deceive people into increasing product sales, defaming politicians or celebrities, and misguiding visitors to stop visiting a place or country. Therefore, it is vital to find automatic methods to detect fake news online. In several past studies, the focus was the English language, but the resource-poor languages have been completely ignored because of the scarcity of labeled corpus. In this study, we investigate this issue in the Urdu language. Our contribution is threefold. First, we design an annotated corpus of Urdu news articles for the fake news detection tasks. Second, we explore three individual machine learning models to detect fake news. Third, we use five ensemble learning methods to ensemble the base-predictors' predictions to improve the fake news detection system's overall performance. Our experiment results on two Urdu news corpora show the superiority of ensemble models over individual machine learning models. Three performance metrics balanced accuracy, the area under the curve, and mean absolute error used to find that Ensemble Selection and Vote models outperform the other machine learning and ensemble learning models.

\section{Introduction}


38 Fake news is also known as deceptive news or misinformation. A news story is a piece of fake

39

40

41

42

43

44

45

46

47

48

49

50

51

52

53

54

55

56

57

58

59

60

61

62

63

64

65

66

67

68

69

70

71

72

73

74

75

76 news if its authenticity is verifiable false, and it intends to mislead the reader. As compared to fake news, the authenticity of legitimate news is verifiable real, and it plans to convey authentic information to the users (Abonizio et al., 2020). Fake news can take on numerous structures including, edited text stories, photoshopped pictures, and unordered video clips. Fake news is similar in appearance to legitimate news, but the aims are different. The aims of spreading fake news are multipurpose, including deceiving readers into benefiting the author, propaganda about a politician to win the election, increased sale of a product by posting fake positive reviews to benefit a businessman, and defame a showbiz star (Monteiro et al., 2018). There are numerous hazardous impacts on our society of the proliferation of fake news. Fake news changes the manner of the individual to interpret and reply to legitimate news. Besides, fake news makes individuals skeptical by destroying consumers' trust in the media by posting fabricated and biased news stories (Agarwal \& Dixit, 2020). Spreading fake news is not a new problem in our time. Before the advent of the internet, fake news was transmitted through face-to-face (oral), radio, newspaper, and television. In recent years with the advent of the computer, the internet, smartphones, websites, news blogs, and social media applications have contributed to transmitting fake news. There are several reasons for spreading fake news through the internet and social media. It requires less cost and time than traditional news media. It is very easy to manipulate legitimate digital news and share the fabricated news story rapidly. Since 2017 , there is a $13 \%$ global increase in social media users (Kaur, Kumar \& Kumaraguru, 2020). Fake news influences different groups of people, products, companies, politicians, showbiz, news agencies, and businessman.

It requires more energy, cost, and time to manually identify and remove fake news or fake reviews from social media. Some previous studies conclude that humans perform poorly than automated systems to separate legitimate news from fake news (Monteiro et al., 2018). For the last few years, machine learning methods' focus is to differentiate between fake and legitimate news automatically. After the U.S. presidential elections in 2015, few popular social media applications like Twitter, Facebook, and Google started to pay attention to design machine learning and natural language processing (NLP) based mechanisms to detect and combat fake news. The remarkable development of supervised machine learning models paved the way for designing expert systems to identify fake news for English, Portuguese (Monteiro et al., 2018; Silva et al., 2020), Spanish (Posadas-Durán et al., 2019), Indonesian (Al-Ash et al., 2019), German, Latin, and Slavic languages (Faustini \& Covões, 2020). A major problem of machine learning models is that different models perform differently on the same corpus. Their performance is sensitive to corpus properties like corpus size, distribution of instances into classes (Pham et al., 2021). For example, the performance of K-nearest neighbor (KNN) depends on the number of nearest points $(\mathrm{k})$ in the dataset. SVM suffers from numerical instability when solving optimization problems (Xiao, 2019). Similarly, the performance of an artificial neural network (ANN) is prone to optimal architecture and tuning its parameters (Pham et al., 2021).

PeerJ Comput. Sci. reviewing PDF | (CS-2020:10:54064:2:0:NEW 6 Feb 2021) 
77 Ensemble learning is considered an efficient technique that can boost the efficiency of individual

78 machine learning models, also called base-models, base-predictors, or base-learners, by

79 aggregating the predictions of these models in some way (Lee et al., 2020). Ensemble learning

80 aims to exploit the diversity of base-predictors to handle multiple types of errors to increase

81 overall performance. Ensemble learning techniques show superior performance in various recent

82 studies about fake news detection. In a recent study, the ensemble learning technique

83 outperformed the four deep learning models including the deep structured semantic model

84 (DSSM) with RNN, intentCapsNet, LSTM model, and capsule neural network (Hakak et al.,

85 2021). In another recent study, (Mahabub, 2020) applied eleven machine learning classifiers

86 including the neural network-based model MLP on a fake news detection corpus. After that three

87 out of eleven machine models were selected to ensemble a voting model. Ensemble voting with

88 soft voting outperformed the other models. (Gutierrez-Espinoza et al., 2020) applied two

89 ensemble methods bagging and boosting with SVM and MLP base-predictors to detect fake

90 reviews detection. Experiments show that boosting with MLP outperforms the other.

91 This can be achieved in numerous ways, including homogenous models with diverse parameters,

92 heterogeneous models, resampling the training corpus, or using different methods to combine

93 predictions of base-predictors (Gupta \& Rani, 2020). Ensemble learning can be of two types:

94 parallel and sequential. In the parallel ensemble, base-predictors are trained independently in

95 parallel. In the sequential ensemble, base-predictors are trained sequentially, where a model

96 attempts to correct its predecessor (Pham et al., 2021). Ensemble learning methods have shown

97 good performance in various applications, including solar irradiance prediction (Lee et al., 2020),

98 slope stability analysis(Pham et al., 2021), natural language processing (Sangamnerkar et al.,

99 2020), malware detection (Gupta \& Rani, 2020), traffic incident detection (Xiao, 2019). In the

100 past, several studies explored machine learning models for fake news detection task in a few

101 languages like Portuguese (Monteiro et al., 2018; Silva et al., 2020), Spanish (Posadas-Durán et

102 al., 2019; Abonizio et al., 2020), Urdu (Amjad et al., 2020; Amjad, Sidorov \& Zhila, 2020),

103 Arabic (Alkhair et al., 2019), Slavic (Faustini \& Covões, 2020; Kapusta \& Obonya, 2020), and

104 English (Kaur, Kumar \& Kumaraguru, 2020; Ozbay \& Alatas, 2020). As compared to machine

105 learning, a few efforts have been made to explore ensemble learning for fake news detection like

106 Indonesian (Al-Ash \& Wibowo, 2018; Al-Ash et al., 2019), English (Kaur, Kumar \&

107 Kumaraguru, 2020; Sangamnerkar et al., 2020). Therefore, this study aims to investigate

108 ensemble learning methods for the fake news detection task.

109 Urdu is the national language of Pakistan and the $8^{\text {th }}$ most spoken language globally, with more

110 than 100 million speakers (Akhter et al., 2020a). Urdu is the South Asian severely resource-poor

111 language. As compared to resource-rich languages like English, a few annotated corpus from

112 very few domains are available for research purposes. Besides, insufficient linguistic resources

113 like stemmers and annotated corpora make the research more challenging and inspired.

114 Particularly in Urdu, studying fake news detection has several challenges. First, unavailability of

115 some sufficient annotated corpus. A recent study (Amjad et al., 2020) proposed an annotated

116 fake news corpus with a few hundred news articles. Experiments on this corpus reveal the poor 
117 performance of machine learning models. Second, labeling a news article as 'fake' or

118 'legitimate' needs experts' opinions, which is time taking. Last, hiring experts in the relevant 119 domains is an expensive task. Therefore, in this study, we design a machine-translated corpus of 120 Urdu news articles translated from English news articles using Google Translate. We followed

121

122

123

124

125

126

127

128

129

130

131

132

133

134

135

136

137

138

139

140

141

142

143

144

145

146

147

148

149

150

151

152

153

154

155

156 the same procedure in the study (Amjad, Sidorov \& Zhila, 2020). Experiments reveal that machine learning models do not perform well on machine-translated corpus compared to the real dataset (Amjad, Sidorov \& Zhila, 2020). Because of the small size, the corpus is not sufficient to make any conclusion about machine learning models' performance. Further, to the best of our knowledge, no study explores ensemble learning models for Urdu fake news detection tasks. Inspired by the work done in other languages, we are investigating the issue of fake news detection in the Urdu language. The major aim of this study is to explore the capability of ensemble learning models in improving fake news predictions in resource-poor language Urdu. Our significant contributions to this study have been summarized below:

- We manually built an annotated news corpus composed of Urdu news articles distributed into legitimate and fake categories.

- We perform several experiments using three diverse traditional machine learning classifiers Naïve Bayes (NB), Decision Tree (DT), and Support Vector Machine (SVM), and five ensemble models, including Stacking, Voting, Grading, Cascade Generalization, and Ensemble Selection, to achieve improved prediction quality relative to conventional individual machine learning models.

- We investigate the performance of our models using three feature sets generated through character-level, word-level, and statistical-based feature selection methods.

- We report experiments of both machine learning and ensemble learning models on two fake news corpora of the Urdu language.

- We comparatively analyze the performance of our models using four performance measures, including balanced accuracy, the area under the curve, time and mean absolute error.

Henceforward this article is organized as follows: Section 2 presents the essential related works. Section 3 provides a brief overview of machine learning and ensemble learning models used in this study. Section 4 will show the architecture of the adopted framework and corpus characteristics. The results of the experiments are comparatively discussed in Section 5. Finally, Section 6 ends the article with conclusions and future directions.

\section{Related Work}

Online social media and instant messaging applications like Facebook, Google, and Twitter are popular these days in talking to your loved ones, expressing your opinion, sharing professional information, or posting news about the subject of interest. Further, it is common to find some information on the internet quickly. Unfortunately, all the information available on social media sites is not accurate and reliable as it is straightforward to manipulate digital information and quickly spread it in the world. Therefore, it is vital to design some accurate, efficient, and reliable automated systems to detect fake news from a large corpus.

Peer) Comput. Sci. reviewing PDF | (CS-2020:10:54064:2:0:NEW 6 Feb 2021) 
157 In the past, numerous machine learning methods have been used to combat fake news. (Monteiro

158

159

160

161

162

163

164

165

166

167

168

169

170

171

172

173

174

175

176

177

178

179

180

181

182

183

184

185

186

187

188

189

190

191

192

193

194

195 et al., 2018) showed that the multi-layer perceptron (MLP) model outperforms the NB and random forest to identify fake news from a large news corpus. The study of (Faustini \& Covões, 2020) concludes that SVM with bag-of-word (BoW) feature outperformed the other on five corpora of three languages Germanic, Latin, and Slavic. A benchmarking study for fake news detection concludes that SVM with linguistic-based word embedding features enables us to classify fake news with high accuracy (Gravanis et al., 2019). A study about Portuguese fake news detection reveals that random forest outperforms the other five machine learning models (Silva et al., 2020). AdaBoost achieves the best performance on a small corpus than the other six models to separate fake news from legitimate news (Amjad et al., 2020). A recent study of fake news detection using supervised artificial intelligence methods shows that the DT is the best model out of twenty-three models (Ozbay \& Alatas, 2020). After analyzing the above studies, we can conclude designing an effective and high-performing system with a careful selection of the machine learning model and the feature selection method.

To overcome individual machine learning models' issues and increase the classification performance, an ensemble of several diverse machine learning models has shown superior performance than individual machine learning in several applications. Xiao applied ensemble techniques with SVM and KNN base learners to detect traffic incidents (Xiao, 2019).

Experiments show the superiority of the ensemble model over individual machine learning models. A recent study about detecting fraud in credit cards concludes that the ensemble approach based on cost-sensitive meta-learning outperforms traditional machine learning models. (Pham et al., 2021) used four ensemble models: boosted trees, bagged trees, random forest, and generalized random forest for slope stability analysis. Experimental results prove the best performance of extreme gradient boosting over other ensemble models and machine learning models. For malware detection, (Gupta \& Rani, 2020) used five base-predictors, and the output of each base-predictor was ranked by calculating and aggregating the output weights. Then using two ensemble techniques Voting and Stacking to rank the output. A higher value ranked by the ensemble technique was the final prediction of the model. After extensive experiments, the study reveals that weighted Voting shows the best performance than Stacking.

Compared to other applications, fake news detection using ensemble learning techniques has very few studies in the past. (Kaur, Kumar \& Kumaraguru, 2020) proposed a multi-level Voting model for the fake news detection task. The study concludes that the proposed model outperforms the other eleven individual machine learning and ensemble learning models. For multiclass fake news detection, (Kaliyar, Goswami \& Narang, 2019; Abonizio et al., 2020) used Gradient Boosting ensemble techniques and compare their performance with several individual machine learning models. Results on multiple corpora show that Gradient Boosting achieves the best performance than any other individual models. A recent study (Huang \& Chen, 2020) proposed a self-adaptive harmony search algorithm to get optimized weights of ensemble models. The proposed algorithm achieved outstanding performance with $99.4 \%$ accuracy. The

PeerJ Comput. Sci. reviewing PDF | (CS-2020:10:54064:2:0:NEW 6 Feb 2021) 
196 Bagging approach to detect fake news showed superior performance than SVM, Multinomial 197 Naïve Bayes, and Random Forest (Al-Ash et al., 2019).

198 English is a resource-rich language, and many linguistic resources are publically available for 199 research purposes. Therefore, several research studies have been performed for the fake news 200 detection task. A study gives a comparison of twenty three publically available datasets of 201 English (Sharma et al., 2019). A recent survey compares different techniques to identify fake 202 news, their credibility detection, and discusses fundamental theories and opportunities (Zhou \& 203 Zafarani, 2020). There is a severe lack of research studies for fake news detection in languages 204 other than English. For the Indonesian language, a study by (Al-Ash et al., 2019) shows that the 205 bagging model outperforms the three individual models: SVM, Random Forest, and Multinomial Naïve Bayes. In (Abonizio et al., 2020) applied three machine learning models (KNN, SVM, and random Forest) and extreme gradient boosting (ensemble model) on five datasets of three languages (English, Portuguese, and Spanish). In another study about fake news detection for Portuguese, random forest shows high accuracy in most of the experience (Silva et al., 2020). Extreme gradient boosting shows the best performance than other individual models. For the Urdu language (Amjad et al., 2020), Adaboost outperforms the other seven machine learning models on a very small corpus. DT improves the classification accuracy for fake news detection for the Slovak language (Kapusta \& Obonya, 2020).

214 The lack of availability of a benchmarked annotated corpora of resource-poor languages are the 215 major problem to investigate and compare the performance of numerous automated methods for 216 fake news detection. Therefore, in several other than English studies, authors designed their corpus by collecting news articles from the internet and other web resources and manually annotating these articles into fake and legitimate news. A list of corpora for several resourcepoor languages is given in Table 1. It can be noticed that all the corpora except 'Fake.Br' are very small in size. Because corpus designing is a costly and time-consuming task, the annotation process requires several experts from various fields to decide about the news article (Amjad, Sidorov \& Zhila, 2020). To date, our proposed corpus Urdu fake news (UFN) is the largest corpus than others.

228

229

230

231

232

233

234

235

\section{Machine Learning and Ensemble Learning Models Machine Learning Models}

This section gives a brief overview of three traditional machine learning models: Naïve Bayes, Decision Tree, and Support Vector Machine. We also described their significant drawbacks, which limit their performance on various tasks.

\section{Naïve Bayes (NB)}

Naïve Bayes used a probabilistic approach based on Bayesian Theorem with two assumptions: 1) all the features are independent of each other in the dataset, and 2) all the features have equal effects. It is simple, popular, and useful for the classification of a large corpus, although the corpus does not hold independence. NB is challenging to interpret for a large corpus, and its assumption about features independence makes its performance poor, especially when the data 
236 distribution is very skewed (Komiya et al., 2011). Several studies have used NB for fake news

237

238

239

240

241

242

243

244

245

246

247

248

249

250

251

252

253

254

255

256

257

258

259

260

261

262

263

264

265

266

267

268

269

270

271

272

273

274

275

detection tasks like for Portuguese (Monteiro et al., 2018) and English (Gravanis et al., 2019).

\section{Decision Tree (DT)}

The decision tree algorithm learns a decision rule inferred from the training data to design a decision tree model. Nodes of the tree represent the feature vectors taken from the text of the news article. Leaf nodes represent the set of possible labels or classes in the corpus. In our case, there are two possible labels: fake and legitimate. The DT predicts the article's label by learning features from the tree's root to one of the leaf nodes. It is simple and easy to interpret because all the information about the model behavior and influential variables is available. Therefore, the DT is also known as a white-box model. Drawbacks of the DT include overfitting and instability, a complex tree for a high-dimensional dataset that is not easy to interpret (Pham et al., 2021). For the fake news detection task, DT has shown good performance for Slovak (Kapusta \& Obonya, 2020), Portuguese (Silva et al., 2020), English (Gravanis et al., 2019; Ozbay \& Alatas, 2020), and Urdu (Amjad et al., 2020) languages.

\section{Support Vector Machine (SVM)}

Support vector machine is a non-parametric machine learning model. The performance of SVM is usually considered suitable for binary classification tasks with high-dimensional data. SVM maps all the features obtained from news articles' text into n-dimensional space where a feature represents the particular coordinate. During training, SVM learns a hyper-plan that best discriminates the features of one class to another. Therefore, SVM is also known as a discriminative classifier. Although SVM performs well with high-dimensionality data, it is difficult to interpret, requires significant computing resources, and faces numerical instability for optimization problems (Pham et al., 2021). SVM shows excellent performance for fake news detection task in several studies of various languages like English (Monteiro et al., 2018; Gravanis et al., 2019), Urdu (Amjad, Sidorov \& Zhila, 2020), Portuguese (Silva et al., 2020), Dutch (Verhoeven \& Daelemans, 2014), Germanic, Latin, and Slavic (Faustini \& Covões, 2020). Ensemble Learning Models

Ensemble learning aggregates the individual machine learning models (base-predictors) to design a superior model to increase overall accuracy by handling the shortcomings of the basepredictors. It is known as the most efficient technique for improving the performance of machine learning models. Nowadays, ensemble learning methods are gaining more popularity than traditional individual machine learning models in numerous classification tasks like fake news detection (Kaur, Kumar \& Kumaraguru, 2020), malware detection (Gupta \& Rani, 2020). Ensemble learning methods fall into two categories: parallel ensemble and sequential ensemble. Both are shown in Figure 1(a) and Figure 1(b). In the parallel ensemble, the base-predictors are trained in parallel on the input corpus. The parallel ensemble has the advantages of simultaneous predictions, utilizing different CPU cores to execute the models simultaneously, and utilizing the characteristics of independence among them. In the sequential ensemble, the base-predictors are trained sequentially where the output of the one base-predictor plus the input corpus is the input to the next base-predictor. In other words, the base-predictors are dependent on each other. The

Peer) Comput. Sci. reviewing PDF | (CS-2020:10:54064:2:0:NEW 6 Feb 2021) 
276

277

278

279

280

281

282

283

284

285

286

287

288

289

290

291

292

293

294

295

296

297

298

299

300

301

302

303

304

305

306

307

308

309

310

311

312

313

314

315

next base-predictor challenge is to try to correct the errors of the previous base-predictor to improve the overall prediction accuracy (Pham et al., 2021). Base-predictors can be homogenous or heterogeneous. In homogenous, a single machine learning model (like DT or NB) is trained in parallel or sequentially, while in heterogeneous different machine learning models (like DT and $\mathrm{NB}$ ) are trained in parallel or sequentially. The ensemble learning method is advantageous if the heterogeneous machine learning models are used as base-predictor (Kittler, Hater \& Duin, 1996). Heterogeneous ensemble learning can be performed using different classifiers with different feature sets, training sets, and evaluation methods. In this section, we provide a brief description of the five ensemble models used in this study.

\section{Stacking}

Stacking model ensembles several base-predictors machine learning models using the stacking method. It was initially proposed by (Ting \& Witten, 1997) and used in several studies for classification tasks like malware detection (Gupta \& Rani, 2020), credit card fraud detection (Olowookere \& Adewale, 2020), and spam detection (Saeed, Rady \& Gharib, 2019). It can perform both classification and regression on data. Base-predictors are trained on input data, and the output of these base-predictors is given to a meta-classifier which makes the final prediction about the class of an input sample. Meta-classifier can be any classifier like Adaboost, Regression, etc. The primary aim of meta-classifier is to learn the optimal weights to combine the predictions of base-predictors and produce better prediction results than individual basepredictor results. Therefore, Stacking reduces the variance and improve the quality of classification. For unseen test articles from the test set, the article is passed to the base-predictor to classify these test articles. Their classification is then passed to stacking-based ensemble learners to make the final classification of that article as either fake or legitimate.

\section{Voting}

Voting is a meta-classifier that combines several base-predictors using different combination rules. Base-predictor can be any machine learning model. Individual base-predictors are trained on training data. The output of these base-predictors is combined using some combination rules like majority voting, minimum or maximum probabilities, or product of probabilities (Kittler, Hater \& Duin, 1996). Majority voting is also known as hard-voting as the class with majority votes is considered the input article's final class. In soft-voting, the final class is a class with the highest probability averaged over the individual predictors (González et al., 2020). Voting method have used in several classification tasks like fake news detection (Kaur, Kumar \& Kumaraguru, 2020), spam detection (Saeed, Rady \& Gharib, 2019), and slope stability analysis (Pham et al., 2021)

\section{Grading}

Grading is an ensemble model originally presented by (Seewald \& Fürnkranz, 2001). Grading is a type of meta-classifier that corrects the graded predictions of base-predictors at the base-level assuming that different base-predictors make different errors. Graded predictions are those predictions that are marked as incorrect or correct predictions by the base-predictor. A metaclassifier is learned for each base-predictor whose basic job is to predict when the base-predictor 
316 will error. These meta-classifiers are trained on a training set constructed from the graded 317 predictions of corresponding base-predictors as new class labels. Grading is different from

318 Stacking that uses the incorrect predictions of base-predictors as the attribute value for meta319 classifier. Several studies show that Grading outperforms the Voting and Stacking ensemble 320 models on classification tasks (Seewald \& Fürnkranz, 2001; González et al., 2020).

321 Cascade Generalization

322 Cascade Generalization belongs to a special Stacking generalization that uses a sequentially

323 layered architecture for combining multiple classifiers. The predictions of several base-predictors

324

325

326

327

328

329

330

331

332

333

334

335

336

337

338

339

340

341

342

343

344

345

346

347

348

349

350

351

352

353

354

355 are used in the next stage for final prediction (Gama \& Brazdil, 2000). An extension of the original data is achieved at each level by inserting new attributes derived from the probability class distribution given by the base-predictors. Cascade Generalization is different from Stacking generalization in that Stacking is parallel, while Cascade is sequential in nature. Because of its sequential nature, intermediate models have access to the original attributes and the low-level models' predictions. Cascade provides the rules to choose the high-level and low-level models for classification. A major problem of the Cascade is that it transforms the feature space into a new high-dimensional feature space (the curse of dimensionality) that sometimes leads to a more difficult learning problem (Gama \& Brazdil, 2000).

\section{Ensemble Selection}

Ensemble selection is a method to construct an ensemble of several machine learning models. It starts with an empty ensemble and adds a model into the ensemble if it increases the performance of the ensemble. This process is repeated up to a specified number of iterations or until all the models have been used (Caruana, Ksikes \& Crew, 2014). Models are added into an ensemble using numerous ways like forwarding selection, backward elimination, and the best model. It stops adding models into the ensemble when the ensemble's performance starts to decrease after achieving the best performance. Ensemble selection allows ensembles to be optimized to performance metrics such as accuracy, cross-entropy, mean precision, or ROC Area (Ballard \& Wang, 2016; Nguyen et al., 2020). In a recent study, Ensemble Selection outperforms the other ensemble models to classify 62 datasets (Nguyen et al., 2020).

\section{Methodology and corpus construction}

The proposed framework for fake news detection consists of four-phases. The first phase describes the procedure to design a corpus of Urdu news articles. The second phase explains the preprocess operations performed on the text of news articles. The third phase shows feature selection or dimensionality reduction approaches. The fourth phase provides the description of individual machine learning models or base-predictors for ensemble models. At last, the fifth phase describes the numerous ensemble learning models used in this study. The architecture with five layers is shown in Figure 2.

\section{Corpus Design}

In this study, we choose two corpora of text news articles of Urdu language for experiments. As Urdu is a resource-poor language, there is no standard corpus available for fake news detection 
356

357

358

359

360

361

362

363

364

365

366

367

368

369

370

371

372

373

374

375

376

377

378

379

380

381

382

383

384

385

386

387

388

389

390

391

392

393

394

395

task to the best of our knowledge. Because of the lack of linguistic resources, the collection of news articles from multiple sources is a tough task. Besides, the annotation process of these news articles based on the articles contents needs expert knowledge, a lot of time, and budget. Therefore, augmented corpus design is the only way to perform research about fake news detection for resource-poor languages. Our first corpus is Urdu Fake News (UFN) augmented corpus. It contains two thousand news articles randomly selected and translated from English language fake news corpus using online Google Translator. The original English corpus contains nearly 8000 news articles. We picked a subset of two thousand articles because 1) manual translation of all the articles in the original corpus is time taking and difficult to perform, 2) English-Urdu translation using Google Translator is not hundred percent accurate and to the best of our knowledge, no study investigates this problem. This is an open research question and can be investigated in future studies. 3) we aim to explore the detection performance of ensemble learning techniques. Several recent studies about fake news detection in Slovak (Kapusta \& Obonya, 2020), Italian (Fornaciari \& Poesio, 2013), and Spanish (Posadas-Durán et al., 2019) used corpora with even less than two thousand news articles (see Table 1). Our second corpus is a small size Bend the Truth (BET) corpus designed and annotated by (Amjad et al., 2020). This corpus contains only 900 original news articles in Urdu. A sample of the Urdu news articles is shown in Table 2. After translation, the Urdu article label was the same as in the English corpus's corresponding article. The final corpus is available online on GitHub in CSV file format. The statistics of both corpora are shown in Table 3. It can be noticed that our designed corpus UFN is larger than the BET corpus based on the total number of articles, size of vocabulary, and the length of the article.

\section{Corpus Preparation and Preprocessing}

Articles in the corpus are in an unstructured format and cannot be processed directly by the machine learning models. We must have to perform a series of operations on the corpus to convert an unstructured corpus into a structured corpus. We have cleaned and processed both corpora's news articles before generating the feature vectors for feature selection. We tokenized the text using space characters. Special characters, email addresses, and website URLs were removed from the text. After cleaning the text, we removed the most frequent and rare words of the Urdu language (also known as stopwords) from the text. The cleaned and the preprocessed articles were converted into numeric feature vectors using the term frequency-inverse document frequency (TF-IDF) method as used in a recent study (Ozbay \& Alatas, 2020). Both corpora were passed through the same number of preprocessing steps.

\section{Feature Selection}

In our experiments, we have performed the experiments using three feature selection methods character tri-grams, bag-of-word (BOW), and information gain (IG). A recent study shows the superiority of the character n-gram method over word-level n-grams in short text classification tasks (i.e. offensive language detection) in Urdu text comments (Akhter et al., 2020b). Character $\mathrm{n}$-gram is a contiguous sequence of characters in the text. In character n-grams, the value of $n$ is

Peer) Comput. Sci. reviewing PDF | (CS-2020:10:54064:2:0:NEW 6 Feb 2021) 
396

397

398

399

400

401

402

403

404

405

406

407

408

409

410

411

412

413

414

415

416

417

418

419

420

421

422

423

424

425

426

427

428

429

430

431

432

433

taken as three, which means the combination of three characters makes a tri-gram feature. From the UFN corpus, 1084 character n-grams, and from the BET corpus $1091 \mathrm{n}$-grams were extracted. BOW is a content-based feature representation in which a news article is represented as a set of words that occur in it at least once. IG measures the goodness of the features in the text. A comparative study concludes that IG is the best feature selection method for documentlevel text classification of Urdu. In our experiments, we have selected the top one thousand IG features from both corpora. 1225 and 1214 BoW features from BET and UFN, respectively.

\section{Heterogeneous Machine Learning Models}

In our experiments, for machine learning classification, we use three individual machine learning models NB, SVM, and DT to detect fake news. All three models are heterogeneous. The working of these models is entirely different from each other. Using character-level n-grams from text articles, these models analyze the article's text and classify it into one of the categories legitimate or fake. Detail description of these machine learning models is given in Section 3.1.

\section{Ensemble Learning Models}

Ensemble classification is usually based on two levels: base-level and ensemble-level. We use three diverse machine learning models, SVM, DT, and NB, as base-predictors at the base-level. Input to these base-predictors is the character-level n-grams extracted from the news articles. Output predictions of these base-predictors are input to ensemble-level models. The basic aim of using the ensemble model is to overcome the base-predictors' shortcomings and improve overall prediction accuracy. We use five ensemble models for ensemble classification, known as Voting, Grading, Stacking, Cascading Generalization, and Ensemble Selection. A brief description of our ensemble models is given in Section 3.2.

\section{Performance Measures}

To compare the performance of individual machine learning models and ensemble learning models, in this study, we employed the three well-known performance measures mean absolute error (MAE), balanced accuracy (BA), and area under the curve (AUC).

\section{Balanced Accuracy (BA)}

Separation of fake news from legitimate news is a binary classification task. A model has to decide about an article, either a legitimate article or a fake article. As the focus of this study is to detect both classes correctly, we used the balanced accuracy performance measure to compare the performance of our models. Balanced accuracy calculates the average of the proportion of corrects of each class individually. Balanced accuracy can be calculated as follows:

$$
\text { Balanced Accuracy }(B A)=\left[\frac{T P}{T P+F P}+\frac{T N}{T N+F N}\right] / 2
$$

\section{Area Under the receiver operating characteristic Curve (AUC)}

Area Under the receiver operating characteristic curve, also known as area under the curve (AUC), is used to estimate the performance of a machine learning model using a single value. AUC provides the probability that the model will rank a randomly chosen positive sample higher than a randomly chosen negative sample. AUC can be calculated by Equation 2. $T P_{\text {rate }}$ is the 
434 ratio of correctly predicted articles as fake articles. It is also known as recall and can be 435 calculated as above in Equation 3. FP rate is the number of legitimate news articles that are 436 misclassified or incorrectly predicted as fake news articles.

$$
\begin{gathered}
A U C=\frac{1+T P_{\text {rate }}-F P_{\text {rate }}}{2} \\
F P_{\text {rate }}=\frac{F P}{F P+T N}
\end{gathered}
$$

437 General rules for categorizing the performance of the machine learning model using AUC are

438

439

440

441

442

443

444

445

446

447

448

449

450

451

452

453

454

455

456

457

458

459

460

461

462

463

464

465

given in Table 4. These rules are used and discussed in (Pham et al., 2021).

\section{Mean Absolute Error (MAE)}

The error refers to the absolute difference between the actual values and the predicted values. MAE measures the average magnitude of the error from a set of predictions made by the model. MAE can be calculated as follows:

$$
\text { Mean Absolute Error }(M A E)=\frac{1}{N} \sum_{j=1}^{n}\left|y_{j}-\hat{y}_{j}\right|
$$

\section{Results}

\section{Experiment Setup}

As mentioned earlier, in this study, three diverse machine learning models NB, DT, and SVM have been used to classify news articles into legitimate and fake classes. We use a well-known data mining tool, WEKA, for experiments. WEKA provides a list of supervised and unsupervised machine learning models, data preprocessing techniques, and various performance evaluation methods. Machine learning models have few parameters, called hyper-parameters, to minimize the difference between training error and testing error. In our experiments, we do not fine-tune the hyper-parameters of all these models. We use the default parameters given in the WEKA as the default parameters have already the best values in most of the cases. We use the J48 algorithm for DT implementation. LibLINEAR algorithm for SVM implementation. We use the same DT, SVM, and NB models as base-predictors for all the ensemble models. For Voting and Stacking, along with the three base-predictors, we use Adaboost as a meta-classifier.

\section{Model training and testing}

For training and validation of individual machine learning models and ensemble models, we use k-fold cross-validation as mentioned in Section 4.1 that both corpora have not been divided into a training subset and testing subset. k-fold cross-validation is a popular choice and used in many past research studies. In our experiments, we use 10-fold cross-validation, where k-1 folds are used for training, and one-fold is used to test the model's prediction performance. This process is repeated ten times to achieve the final performance score.

\section{Results and discussion of machine learning models}


466 The experiment results achieved using 10-fold cross-validation from individual machine learning 467 models are shown in Table 5. We compare the performance using BA, AUC, MAE, and time. A 468 close observation of the results reveals that SVM outperforms the other for all corpora 469 performance metrics. A model is considered an accurate classifier if its balanced accuracy is higher than the other models. The BA metric shows that SVM outperforms the others on the UFN corpus. SVM achieves BA scores $81.6 \%, 86.7 \%$, and $87.3 \%$ using tri-gram, BoW, and IG features, respectively. SVM also outperforms the others on the BET corpus. It achieves 76.3, 62.7, and 62.4 using tri-gram, BoW, and IG features, respectively. IG feature outperforms the others and achieves the maximum BA score $87.3 \%$ on large UFN corpus while tri-gram approach achieves maximum BA scores of $76.3 \%$ on BET corpus. With the lowest balanced accuracy scores, NB shows the worst performance. It is noticed that SVM has higher accuracy at UFN than BET. The size of the UFN corpus, in terms of the number of articles and vocabulary size, is almost double the of BET, and SVM is considered a good model for the classification of high-dimensional feature space (Faustini \& Covões, 2020). Similarly, AUC scores of the SVM model are the highest score than DT and NB on both corpora. SVM achieves $87.3 \%$ and $76.3 \%$ AUC metric values on UFN and BET corpora, respectively. Here, again IG proves the best feature selection method for UFN while tri-gram on BET corpus as SVM achieves the maximum AUC scores on IG and tri-gram features. Further, as per the rules of Table 4, a comparison of AUC scores of all the models concludes that the performance of SVM on UFN is excellent $(0.8 \leq A U C<0.9)$ on all the features. On the BET corpus, SVM performance is only acceptable $(0.7 \leq A U C<0.8)$ on tri-gram features. The performance of DT and NB is just acceptable.

From Table 5, it can be seen that in terms of MAE, the prediction error of SVM is the lowest than others. SVM achieves the lowest MAE score $12.7 \%$ with IG on UFN while $23.5 \%$ with trigram on BET corpus. The highest MAE values of NB proves its worst performance to detect Urdu fake news. A model is considered efficient if it takes minimum time than other models to build the model on some corpus. Again, SVM takes a minimum time of 0.15 on BOW and 0.17 seconds on IG to build the model on UFN and BET. DT takes the highest time on all features to build the model for both corpora. Further, it is notable that all the models perform betters on our designed machine-translated UFN corpus than the original news article's corpus BET. It shows that Google API translated text, preprocessing methods, and feature selection methods all together improve the classification accuracy of our models to detect fake news. Therefore, after analyzing the results, we conclude that SVM is a more accurate, reliable, efficient, and robust classification model among the three models to detect Urdu text's fake news articles.

\section{Results and discussion of ensemble models}

The values of four evaluation metrics balanced accuracy, the area under curve, mean absolute error, and time achieved by five ensemble models on both corpora are given in Table 6 . Time and MEA are the two metrics whose minimum values are required by the models. The other two metrics balanced accuracy and AUC, whose maximum values are required to achieve by a model. For the time metric, it is visible that Voting takes the minimum time than other models to 
506 build the model on the input corpus. Voting takes 11.52 seconds and 3.12 seconds to build the 507 model on UFN and BET corpora, respectively. As the size of the BET model is very small, the

508 Voting takes the minimum time to build the model than all the other models. It can also be 509 noticed that the minimum time taken by Voting to build a model on both corpora is using tri510 gram. It shows the efficiency of the tri-gram method over IG and BoW to build a model.

511 For the MAE metric, again, the Voting model achieves the minimum values than others on both 512 corpora, which shows that magnitude of the error is significantly less in predicting the labels of 513 both types of articles. The average magnitude error of the Voting model is $18.41 \%$ on tri-gram 514 and $10.7 \%$ on IG for BET and UFN, respectively. It means that IG is a good feature selection 515 method over other methods on large size UFN corpus while tri-gram is good for small size BET 516 corpus.

517 To estimate an ensemble model's performance and decide whether a model's performance is 518 acceptable or not, we use a performance ranking metric AUC. On the BET corpus, only $519 A U C \geq 90$ is achieved by the Ensemble Selection model over the tri-gram feature method. With 520 IG and BoW features the AUC scores of all the other models are $A U C<75$ which means the 521 performance of these models is acceptable. On UNF corpus, Cascade Generalization achieves 522 outstanding performance to detect fake news with BoW and IG (see Table 4). It achieves 92.0\% 523 and $92.7 \%$ AUC scores $(A U C \geq 90)$ for BoW and IG methods. Cascade Generalization with $52486.8 \%$ AUC score categorizes its performance ranking to excellent. Again, Ensemble Selection

525

526

527

528

529

530

531

532

533

534

535

536

537

538

539

540

541

542

543

544 achieves the best AUC score using IG on UFN while Cascade Generalization achieves the best AUC using tri-gram features on BET.

As we are interested to know the performance of a model to predict both labels ('fake', 'legitimate) correctly in the corpus, we use balanced accuracy. The maximum BA achieved by a model means that the model is performing more accurately than others to distinguish fake articles from legitimate articles. Experiment results reveal that Ensemble Selection and Voting outperform the other models on BET corpus and UFN corpora. Ensemble Selection achieves the maximum $83.3 \%$ BA on BET corpus using the tri-gram feature On the UFN corpus, the Voting model significantly outperforms the other four ensemble models and achieves an $89.3 \%$ BA score using the IG feature. Again, it is noticed that IG outperforms the other methods on UFN while tri-gram outperforms the other feature selection methods on the BET corpus.

Voting model ensemble the numerous base-predictors using some ensemble rule. The four popular rules are majority voting, the product of probabilities, minimum probabilities, and maximum probabilities. By impressive Voting performance on both corpora using balanced accuracy, MAE, and Time metrics, as given in Table 6 and discussed above, we further investigate its performance using different ensemble rules. The mean absolute error values achieved by each ensemble rule is shown in Figure 3. We conclude that the minimum probabilities rule is impressive to ensemble the predictions of the base-predictors at it achieves the lowest error values on both corpora. The vote model achieves $16.74 \%$ and $18.41 \% \mathrm{MAE}$ 
545 scores on UFN and BET corpora. Hence, in our experiments, minimum probabilities, and

546 product of probabilities, both rules perform the same on both corpora.

547 Performance comparison of machine learning and ensemble learning models

548 It is important to know the difference in the performance of ensemble models and individual

549 machine learning models.

550 A summary of the results achieved by the best ML and EL model with the best feature selection

551 method is given in Table 7. Comparative analysis of the results shows that machine learning

552 models are efficient than EL models and take less time to build a model on both corpora. SVM

553 takes a minimum time of less than a second to build the model on both corpora. Among the EL

554 models, Voting is efficient and takes 11.52 and 3.12 seconds on UFN and BET. But Voting is

555 much costly than SVM. It is because of the multiple base-predictors in the EL model. EL model

556 combines the three heterogeneous ML models and then the final Voting model predicts the final

557 label based on the prediction of the base-models.

558 For error analysis, MAE values show that EL models have the lowest values of MAE than

559 individual ML models. Again SVM outperforms the NB and DT by achieving minimum MAE

560 scores on both corpora. On the other side, Voting outperforms the other EL models on both

561 corpora. The lowest score of MAE for EL models means that these models are more accurate in

562 fake news detection. EL models reduce MAE at two levels: at the base-predictors level and

563 ensemble-level. Voting takes the advantage of MAEs of its base-predictor. It reduces the MAE

564 scores of its three base-predictors using minimum probability to predict the final class.

565 SVM achieves maximum scores of AUC $87.3 \%$ and $76.3 \%$ on UFN and BET. AUC scores rank

566 SVM predictions to excellent on UFN and acceptable on BET. Cascade Learning and Ensemble

567 Learning achieve $92.7 \%$ and $91.0 \%$ AUC scores on UFN and BET. It categorizes the detection

568 performance of both models as outstanding. SVM outperforms the other ML models and it

569 achieves the maximum BA scores. SVM achieves $87.3 \%$ BA on UFN and $76.3 \%$ on BET. From

570 EL models, Voting achieves 89.3\% BA and outperforms the other EL and ML models on the

571 UFN corpus. On BET corpus, Ensemble Selection models produce $83.3 \%$ BA that is the

572 maximum BA among all the models.

573 The comparison of EL and ML methods using three feature selection methods is interesting

574 valuable. SVM shows the best performance among the three ML models on small and large

575 corpora. SVM achieves the best scores in all the performance measures. Character tri-gram

576 works well on small size corpus BET while IG works well on large size corpus UFN to boost

577 SVM performance. Voting performance is the best performance among EL models using Time

578 and MAE performance measures on both corpora. Ensemble Selection is good at small corpus

579 BET on two performance measures. IG feature works well with Voting to predict the class of a

580 news article on UFN while tri-gram is the best with Voting and Ensemble Learning. Further, it

581 can be seen that the IG feature works well on large size corpus while character tri-gram is good

582 on small size corpus.

583

584

\section{Conclusions}


585 Fake news detection through ensemble models is the most prominent topic of machine learning. 586 If the traditional machine learning models are used for fake news detection task, the performance 587 is not encouraging because their performance is limited to corpus characteristics. In this study, 588 we deliberately choose ensemble methods to classify fake and legitimate news articles of the 589 Urdu language. First, we use three machine learning models to classify two fake news corpora. 590 Our experiments on two Urdu news corpora conclude that the individual machine learning model 591 SVM outperforms the DT and NB on both corpora. SVM achieves the best scores of balanced 592 accuracy and AUC and the minimum score of MAE. Second, we use five ensemble models for 593 the same task.

594 We find that ensemble models with three base-predictors DT, NB, and SVM, Ensemble

595

596

597

598

599

600

601

602

603

604

605

606

607

608

609

610

611

612

613

614

615

616

617

618

619

620

621

622

623

624

625

626

627

Selection, and Vote models outperform the other on BET and UFN corpora, respectively. After the analysis of MAE, AUC, time, and BA values, we conclude that Voting with minimum probability is the best EL model for the fake news detection task. IG feature works well with large size corpus while character tri-gram works well on small size corpora.

This study has several limitations that need to be addressed in future studies. The proposed corpus UFN still needs to grow by adding more news articles to it. We used online Google translation API in English-to-Urdu translation and we believe that translation accuracy is not a hundred percent. A study is vital in the future to explore the translation accuracy and quality of various translation APIs like Baidu, Google, etc. The potential of deep learning models also can be explored to detect fake news for Urdu. Further, we also hope to design another multilingual fake news corpus of English and Urdu news articles.

\section{References}

Abonizio HQ, de Morais JI, Tavares GM, Junior SB. 2020. Language-independent fake news detection: English, Portuguese, and Spanish mutual features. Future Internet 12:1-18. DOI: 10.3390/FI12050087.

Agarwal A, Dixit A. 2020. Fake News Detection: An Ensemble Learning Approach. Proceedings of the International Conference on Intelligent Computing and Control Systems, ICICCS 2020:1178-1183. DOI: 10.1109/ICICCS48265.2020.9121030.

Akhter MP, Jiangbin Z, Naqvi IR, Abdelmajeed M, Fayyaz M. 2020a. Exploring deep learning approaches for Urdu text classification in product manufacturing. Enterprise Information Systems:1-26. DOI: 10.1080/17517575.2020.1755455.

Akhter MP, Jiangbin Z, Naqvi IR, Abdelmajeed M, Sadiq MT. 2020b. Automatic Detection of Offensive Language for Urdu and Roman Urdu. IEEE Access 8:91213-91226. DOI: 10.1109/ACCESS.2020.2994950.

Al-Ash HS, Putri MF, Mursanto P, Bustamam A. 2019. Ensemble Learning Approach on Indonesian Fake News Classification. In: 2019 3rd International Conference on Informatics and Computational Sciences (ICICoS). 1-6. DOI: 10.1109/ICICoS48119.2019.8982409.

Al-Ash HS, Wibowo WC. 2018. Fake News Identification Characteristics Using Named Entity Recognition and Phrase Detection. In: 2018 10th International Conference on Information Technology and Electrical Engineering (ICITEE). 12-17. DOI: 10.1109/ICITEED.2018.8534898.

Alkhair M, Meftouh K, Smaili K, Othman N. 2019. An Arabic Corpus of Fake News: Collection,

Peer) Comput. Sci. reviewing PDF | (CS-2020:10:54064:2:0:NEW 6 Feb 2021) 
628

629

630

631

632

633

634

635

636

637

638

639

640

641

642

643

644

645

646

647

648

649

650

651

652

653

654

655

656

657

658

659

660

661

662

663

664

665

666

667

668

669

670

671

672

673
Analysis and Classification. In: 292-302. DOI: 10.1007/978-3-030-32959-4_21.

Amjad M, Sidorov G, Zhila A. 2020. Data Augmentation using Machine Translation for Fake News Detection in the Urdu Language. Proceedings of The 12th Language Resources and Evaluation Conference:2530-2535.

Amjad M, Sidorov G, Zhila A, Gómez-Adorno H, Voronkov I, Gelbukh A. 2020. "Bend the truth": Benchmark dataset for fake news detection in urdu language and its evaluation. Journal of Intelligent \& Fuzzy Systems:1-13. DOI: 10.3233/jifs-179905.

Ballard C, Wang W. 2016. Dynamic ensemble selection methods for heterogeneous data mining. In: 2016 12th World Congress on Intelligent Control and Automation (WCICA). IEEE, 1021-1026. DOI: 10.1109/WCICA.2016.7578244.

Caruana R, Ksikes A, Crew G. 2014. Ensemble selection from libraries of models. In: Proceedings of the twenty-first international conference on Machine learning.

Faustini PHA, Covões TF. 2020. Fake news detection in multiple platforms and languages. Expert Systems with Applications 158:113503. DOI: 10.1016/j.eswa.2020.113503.

Fornaciari T, Poesio M. 2013. Automatic deception detection in Italian court cases. Artificial Intelligence and Law 21:303-340. DOI: 10.1007/s10506-013-9140-4.

Gama J, Brazdil P. 2000. Cascade Generalization. Machine Learning 41:315-343. DOI: 10.1023/A:1007652114878.

González S, García S, Del Ser J, Rokach L, Herrera F. 2020. A practical tutorial on bagging and boosting based ensembles for machine learning: Algorithms, software tools, performance study, practical perspectives and opportunities. Information Fusion 64:205-237. DOI: 10.1016/j.inffus.2020.07.007.

Gravanis G, Vakali A, Diamantaras K, Karadais P. 2019. Behind the cues : A benchmarking study for fake news detection. Expert Systems With Applications 128:201-213. DOI: 10.1016/j.eswa.2019.03.036.

Gupta D, Rani R. 2020. Improving malware detection using big data and ensemble learning. Computers and Electrical Engineering 86:106729. DOI: 10.1016/j.compeleceng.2020.106729.

Gutierrez-Espinoza L, Abri F, Namin AS, Jones KS, Sears DRW. 2020. Fake Reviews Detection through Ensemble Learning. arXiv:1-8.

Hakak S, Alazab M, Khan S, Gadekallu TR, Maddikunta PKR, Khan WZ. 2021. An ensemble machine learning approach through effective feature extraction to classify fake news. Future Generation Computer Systems 117:47-58. DOI: 10.1016/j.future.2020.11.022.

Huang YF, Chen PH. 2020. Fake news detection using an ensemble learning model based on Self-Adaptive Harmony Search algorithms. Expert Systems with Applications 159:113584. DOI: $10.1016 /$ j.eswa.2020.113584.

Kaliyar RK, Goswami A, Narang P. 2019. Multiclass Fake News Detection using Ensemble Machine Learning. In: Proceedings of the 2019 IEEE 9th International Conference on Advanced Computing, IACC 2019. 103-107. DOI: 10.1109/IACC48062.2019.8971579.

Kapusta J, Obonya J. 2020. Improvement of misleading and fake news classification for flective languages by morphological group analysis. Informatics 7 . DOI: 10.3390/informatics7010004.

Kaur S, Kumar P, Kumaraguru P. 2020. Automating fake news detection system using multilevel voting model. Soft Computing 24:9049-9069. DOI: 10.1007/s00500-019-04436-y.

Kittler J, Hater M, Duin RPW. 1996. Combining classifiers. Proceedings - International Conference on Pattern Recognition 2:897-901. DOI: 10.1109/ICPR.1996.547205. 
674 Komiya K, Sato N, Fujimoto K, Kotani Y. 2011. Negation naive bayes for categorization of 675 product pages on the web. International Conference Recent Advances in Natural Language $676 \quad$ Processing, RANLP:586-591.

677

678

679

680

Lee J, Wang W, Harrou F, Sun Y. 2020. Reliable solar irradiance prediction using ensemble learning-based models: A comparative study. Energy Conversion and Management 208:112582. DOI: 10.1016/j.enconman.2020.112582.

Mahabub A. 2020. A robust technique of fake news detection using Ensemble Voting Classifier and comparison with other classifiers. SN Applied Sciences 2:525. DOI: 10.1007/s42452020-2326-y.

682

683

684

685

686

687

688

689

690

691

692

693

694

695

696

697

698

699

700

701

702

703

704

705

706

707

708

709

710

711

712

713

714

715

716

717

718

719

Monteiro RA, Santos RLS, Pardo TAS, de Almeida TA, Ruiz EES, Vale OA. 2018. Contributions to the Study of Fake News in Portuguese: New Corpus and Automatic Detection Results. In: Lecture Notes in Computer Science (including subseries Lecture Notes in Artificial Intelligence and Lecture Notes in Bioinformatics). Springer International Publishing, 324-334. DOI: 10.1007/978-3-319-99722-3_33.

Nguyen TT, Luong AV, Dang MT, Liew AW-C, McCall J. 2020. Ensemble Selection based on Classifier Prediction Confidence. Pattern Recognition 100:107104. DOI: 10.1016/j.patcog.2019.107104.

Olowookere TA, Adewale OS. 2020. A framework for detecting credit card fraud with costsensitive meta-learning ensemble approach. Scientific African 8. DOI: 10.1016/j.sciaf.2020.e00464.

Ozbay FA, Alatas B. 2020. Fake news detection within online social media using supervised artificial intelligence algorithms. Physica A: Statistical Mechanics and its Applications 540:123174. DOI: 10.1016/j.physa.2019.123174.

Pham K, Kim D, Park S, Choi H. 2021. Ensemble learning-based classification models for slope stability analysis. Catena 196:104886. DOI: 10.1016/j.catena.2020.104886.

Posadas-Durán J-P, Gómez-Adorno H, Sidorov G, Escobar JJM. 2019. Detection of fake news in a new corpus for the Spanish language. Journal of Intelligent \& Fuzzy Systems 36:48694876. DOI: $10.3233 / J I F S-179034$.

Saeed RMK, Rady S, Gharib TF. 2019. An ensemble approach for spam detection in Arabic opinion texts. Journal of King Saud University - Computer and Information Sciences. DOI: 10.1016/j.jksuci.2019.10.002.

Sangamnerkar S, Srinivasan R, Christhuraj MR, Sukumaran R. 2020. An ensemble technique to detect fabricated news article using machine learning and natural language processing techniques. 2020 International Conference for Emerging Technology, INCET 2020:1-7. DOI: 10.1109/INCET49848.2020.9154053.

Seewald AK, Fürnkranz J. 2001. An evaluation of grading classifiers. Lecture Notes in Computer Science (including subseries Lecture Notes in Artificial Intelligence and Lecture Notes in Bioinformatics) 2189:115-124. DOI: 10.1007/3-540-44816-0_12.

Sharma K, Qian F, Jiang H, Ruchansky N, Zhang M, Liu Y. 2019. Combating Fake News: A Survey on Identification and Mitigation Techniques. ACM Trans. Intell. Syst. Technol. 10. DOI: $10.1145 / 3305260$.

Silva RM, Santos RLS, Almeida TA, Pardo TAS. 2020. Towards automatically filtering fake news in Portuguese. Expert Systems with Applications 146:113199. DOI: 10.1016/j.eswa.2020.113199.

Ting KM, Witten IH. 1997. Stacking bagged and dagged models. In: Fisher DH ed. Proc. of ICML'97. 367-375. 
720

721

722

723

724

725

726

727

728
Verhoeven B, Daelemans W. 2014. CLiPS stylometry investigation (CSI) corpus: A Dutch corpus for the detection of age, gender, personality, sentiment and deception in text. In: Proceedings of the 9th International Conference on Language Resources and Evaluation, LREC 2014. 3081-3085.

Xiao J. 2019. SVM and KNN ensemble learning for traffic incident detection. Physica A: Statistical Mechanics and its Applications 517:29-35. DOI: 10.1016/j.physa.2018.10.060. Zhou X, Zafarani R. 2020. A Survey of Fake News: Fundamental Theories, Detection Methods, and Opportunities. ACM Comput. Surv. 53. DOI: 10.1145/3395046. 
Figure 1

Parallel vs. Sequential Ensemble

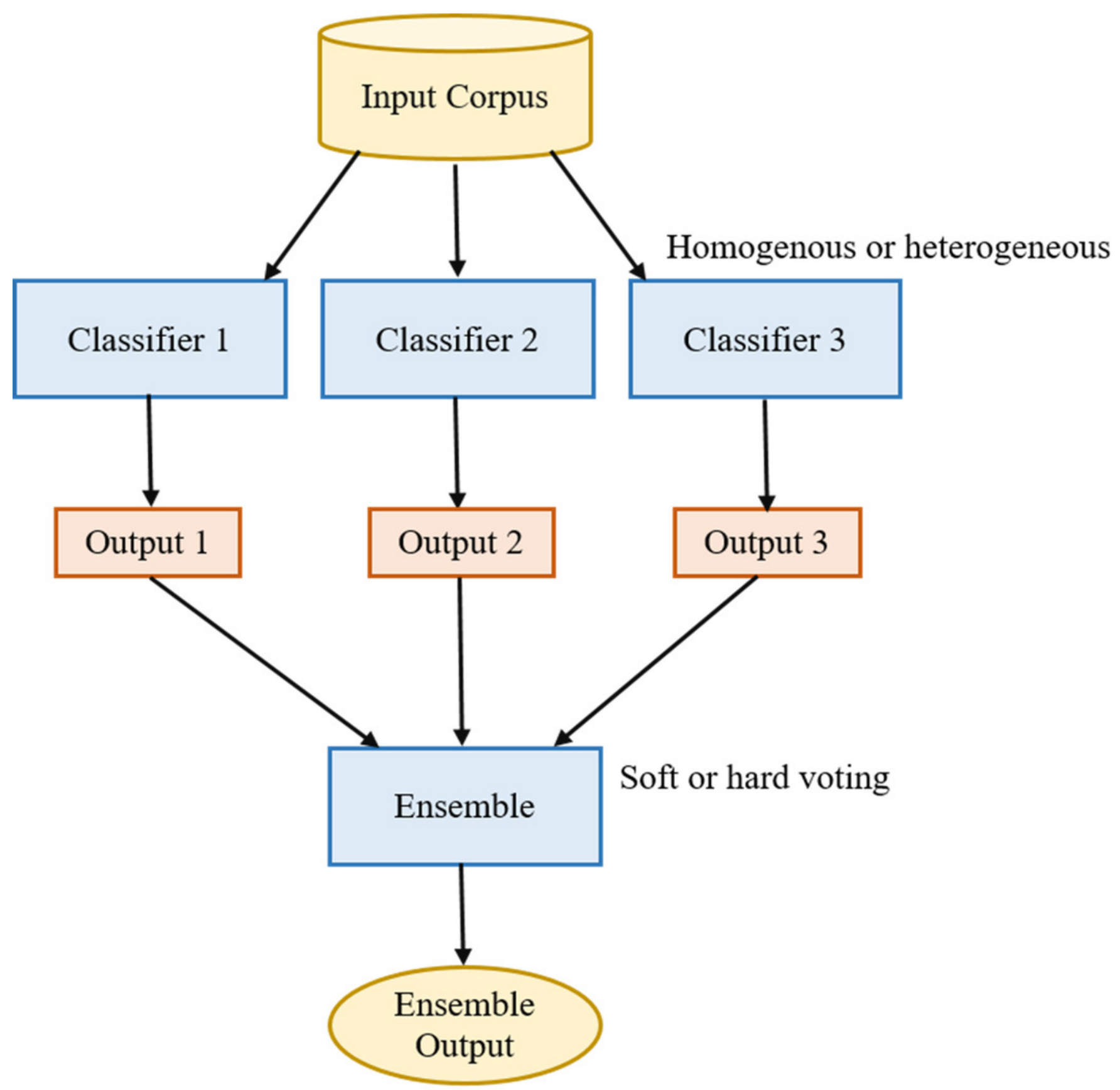


Figure 2

The architecture of the proposed framework used for fake news detection

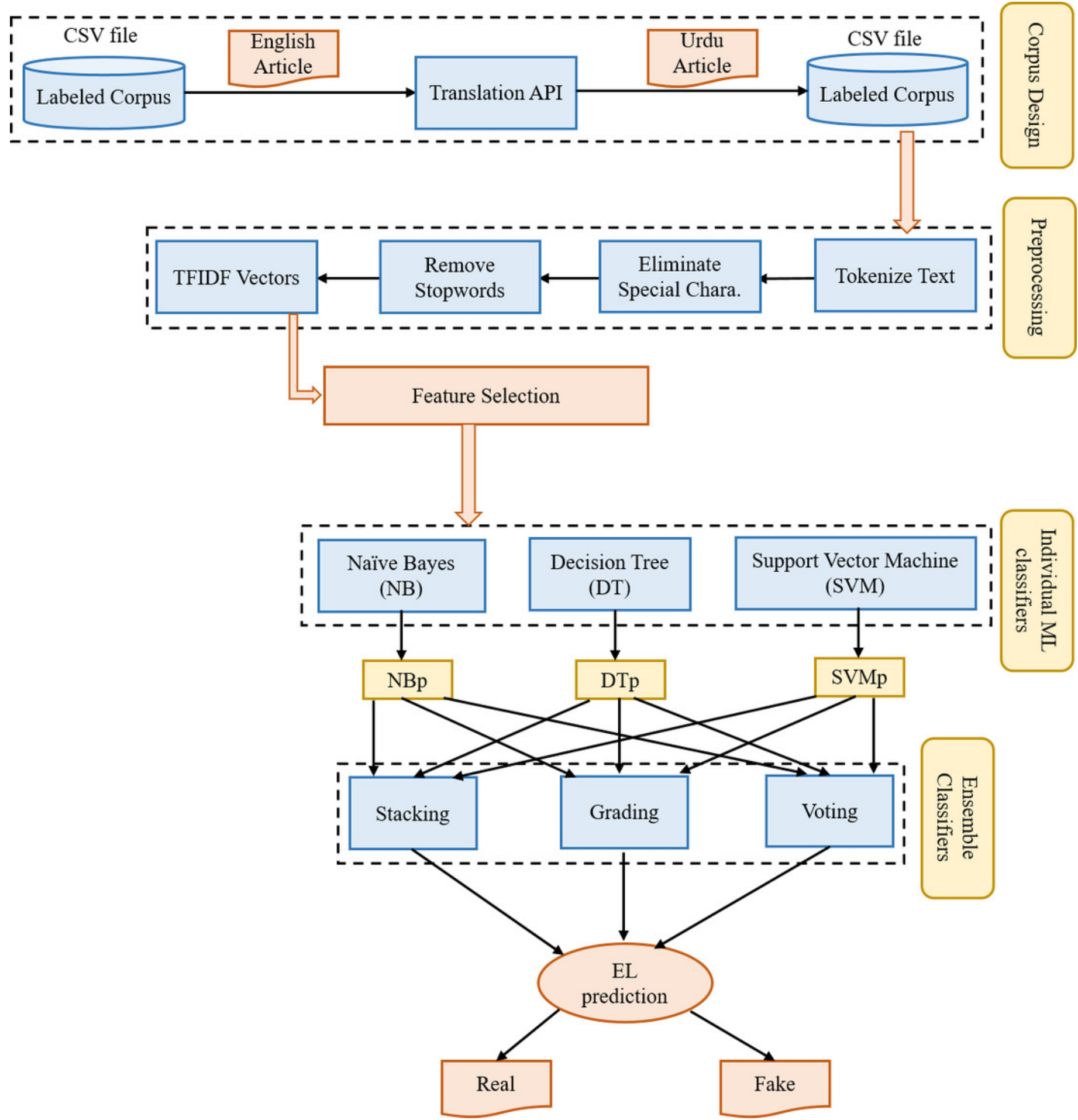


Figure 3

Performance of Voting model using numerous ensemble rules

35

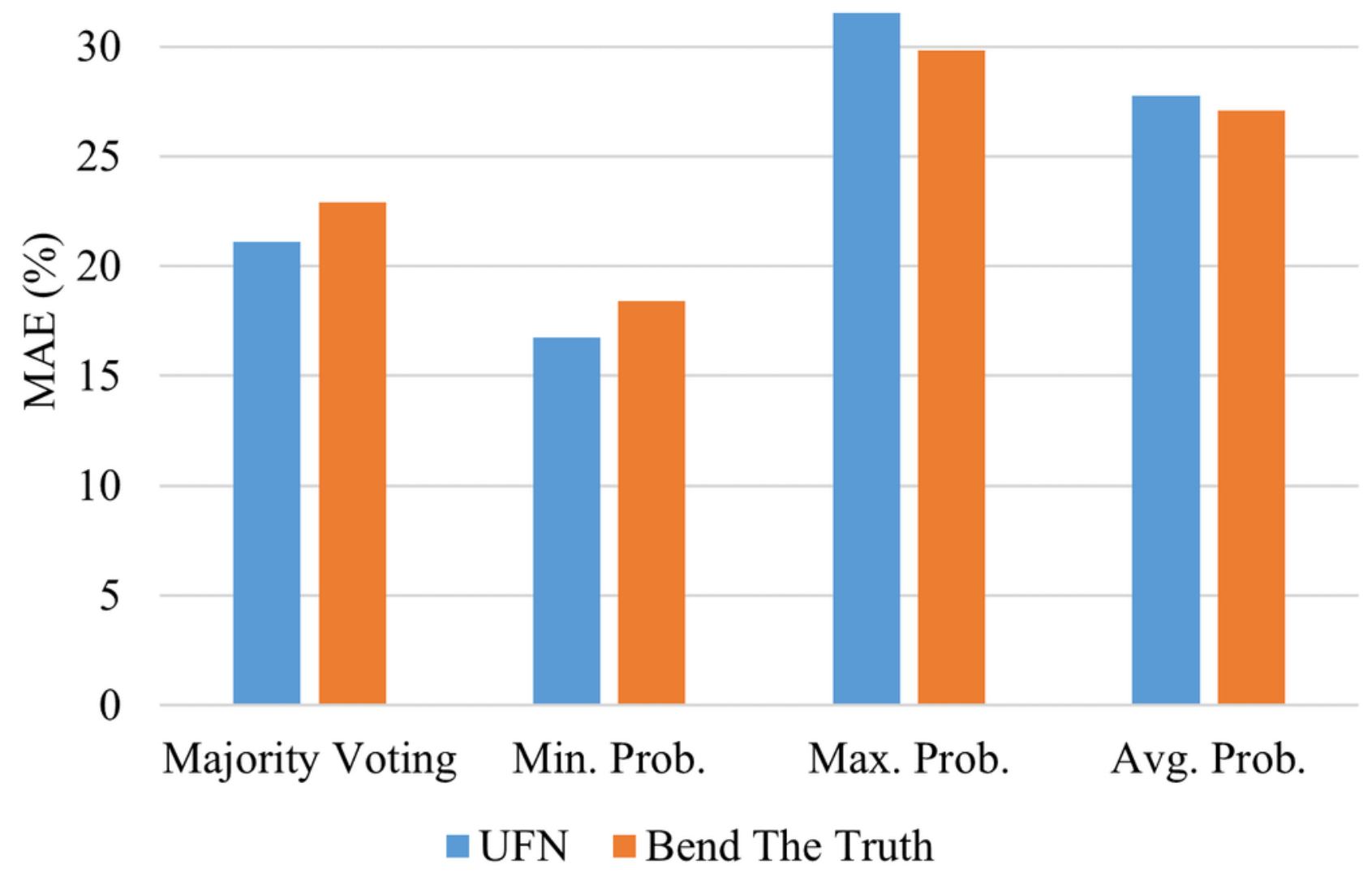




\section{Table $\mathbf{1}$ (on next page)}

Resource-poor language corpora for fake news detection 


\section{Table 1:}

2 Resource-poor language corpora for fake news detection

\begin{tabular}{|l|l|l|l|l|}
\hline Corpus & Language & Legitimate & Fake & References \\
\hline-- & Chinese & 131 & 187 & (Zhang et al., 2009) \\
\hline $\begin{array}{l}\text { Slovak National } \\
\text { Corpus }\end{array}$ & Slovak & 80 & 80 & (Kapusta \& Obonya, 2020) \\
\hline DECOUR & Italian & 1202 & 945 & (Fornaciari \& Poesio, 2013) \\
\hline-- & $\begin{array}{l}\text { English and } \\
\text { Spanish }\end{array}$ & 100 & 100 & (Pérez-Rosas et al., 2018) \\
\hline CSI & Dutch & 270 & 270 & $\begin{array}{l}\text { (Verhoeven \& Daelemans, } \\
\text { 2014) }\end{array}$ \\
\hline Fake.Br & Portuguese & 3600 & 3600 & (Silva et al., 2020) \\
\hline TwitterBR & Brazilian & $\underline{4589}$ & $\underline{4392}$ & (Faustini \& Covoes, 2019) \\
\hline Btvlifestyle & Bulgarian & $\underline{69}$ & $\underline{69}$ & $\begin{array}{l}\text { (Hardalov, Koychev \& } \\
\text { Nakov, 2016) }\end{array}$ \\
\hline-- & Bangla & $\underline{1548}$ & $\underline{993}$ & (Hussain et al., 2020) \\
\hline Spanish Fake News & Spanish & 491 & 480 & (Posadas-Durán et al., 2019) \\
\hline Bend the Truth & Urdu & 500 & 400 & (Amjad et al., 2020) \\
\hline MT & Urdu & 400 & 400 & $\begin{array}{l}\text { (Amjad, Sidorov \& Zhila, } \\
\text { 2020) }\end{array}$ \\
\hline UFN & Urdu & 1032 & 968 & Our corpus \\
\hline
\end{tabular}




\section{Table 2 (on next page)}

Samples of the legitimate and fake news 


\section{Table 2:}

2 Samples of the legitimate and fake news

\begin{tabular}{|c|c|}
\hline Legitimate & Fake \\
\hline 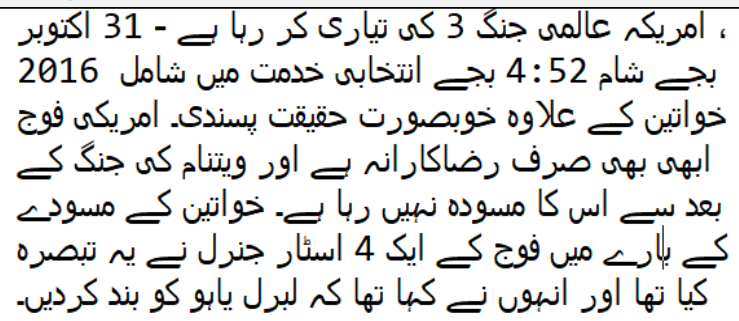 & 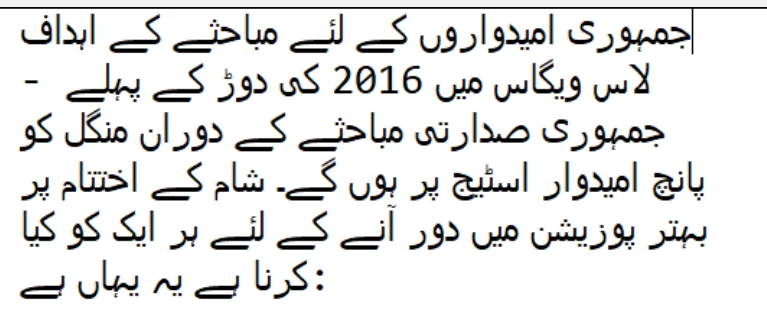 \\
\hline 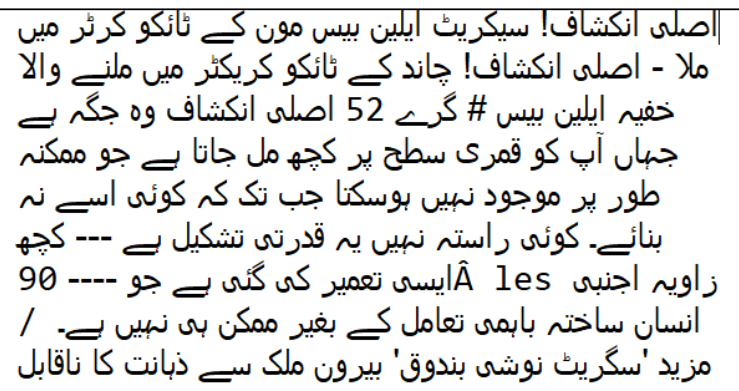 & 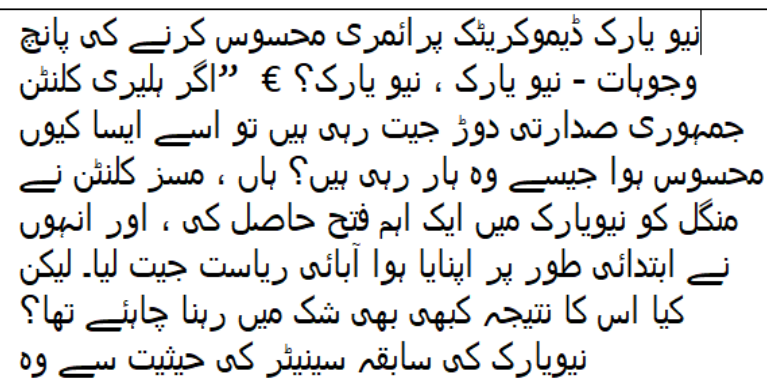 \\
\hline
\end{tabular}




\section{Table 3 (on next page)}

The statistical description of the Urdu language corpora for fake news detection 
1 Table 3:

2 The statistical description of the Urdu language corpora for fake news detection

\begin{tabular}{|l|l|l|l|l|}
\hline Corpus & \multicolumn{3}{|l|}{ Bend the Truth } & \multicolumn{2}{l|}{ Urdu Fake News } \\
\hline Labels & Fake & Legitimate & Fake & Legitimate \\
\hline Vocabulary & $1,84,023$ & $1,20,394$ & $11,47,547$ & $9,54,254$ \\
\hline Minimum Length Article & 57 & 59 & 25 & 25 \\
\hline Maximum Length Article & 2159 & 1153 & 7045 & 6068 \\
\hline Total Article & 400 & 500 & 968 & 1032 \\
\hline
\end{tabular}

3 


\section{Table 4 (on next page)}

Rules for classifying the discrimination using AUC 
1 Table 4:

2 Rules for classifying the discrimination using AUC

\begin{tabular}{|l|l|}
\hline AUC values & Classifier categories \\
\hline$A U C<0.5$ & No Discrimination \\
\hline $0.7 \leq A U C<0.8$ & Acceptable \\
\hline $0.8 \leq A U C<0.9$ & Excellent \\
\hline $0.9 \leq A U C$ & Outstanding \\
\hline
\end{tabular}

3 


\section{Table 5 (on next page)}

The evaluation metrics values of supervised individual machine learning models 
1 Table 5:

2 The evaluation metrics values of supervised individual machine learning models

\begin{tabular}{|l|l|l|l|l|l|l|l|l|}
\hline \multirow{2}{*}{ Ensemble Models } & \multicolumn{3}{|l|}{ BA } & \multicolumn{1}{l|}{ AUC } & \multicolumn{2}{l|}{ MAE } & \multicolumn{2}{l|}{ Time } \\
\cline { 2 - 10 } & UFN & BET & UFN & BET & UFN & BET & UFN & BET \\
\hline Naïve Bayes & 64.8 & 67.4 & 69.4 & 75.5 & 35.5 & 34.2 & $\mathbf{0 . 9 4}$ & $\mathbf{0 . 3 2}$ \\
\hline Support Vector Machine & $\mathbf{8 1 . 6}$ & $\mathbf{7 6 . 3}$ & $\mathbf{8 1 . 5}$ & $\mathbf{7 6 . 3}$ & $\mathbf{1 8 . 5}$ & $\mathbf{2 3 . 5}$ & 2.56 & 0.67 \\
\hline Decision Tree & 71.2 & 76.1 & 69.8 & 73.1 & 29.2 & 23.5 & 8.02 & 2.19 \\
\hline & \multicolumn{8}{|c|}{ BoW } \\
\hline Naïve Bayes & 75.3 & 56.3 & 80.1 & 61.9 & 24.6 & 43.4 & 0.73 & $\mathbf{0 . 4 1}$ \\
\hline Support Vector Machine & $\mathbf{8 6 . 7}$ & $\mathbf{6 2 . 7}$ & $\mathbf{8 6 . 7}$ & $\mathbf{6 2 . 7}$ & $\mathbf{1 3 . 3}$ & $\mathbf{3 7 . 1}$ & $\mathbf{0 . 1 5}$ & 0.8 \\
\hline Decision Tree & 76.6 & 55.6 & 76.6 & 58.4 & 23.8 & 43.5 & 10.96 & 4.39 \\
\hline & \multicolumn{8}{|c|}{ IG } \\
\hline Naïve Bayes & 75.3 & 56.7 & 80.5 & $\mathbf{6 3 . 5}$ & 24.4 & 42.2 & 0.59 & 0.24 \\
\hline Support Vector Machine & $\mathbf{8 7 . 3}$ & $\mathbf{6 2 . 4}$ & $\mathbf{8 7 . 3}$ & 62.4 & $\mathbf{1 2 . 7}$ & $\mathbf{3 7 . 7}$ & $\mathbf{0 . 1 9}$ & $\mathbf{0 . 1 7}$ \\
\hline Decision Tree & 76.8 & 57.0 & 76.8 & 61.5 & 23.6 & 41.6 & 10.28 & 3.56 \\
\hline
\end{tabular}

3 


\section{Table 6(on next page)}

Evaluation metrics for supervised ensemble models using both corpora 
1 Table 6:

2 Evaluation metrics for supervised ensemble models using both corpora

\begin{tabular}{|c|c|c|c|c|c|c|c|c|}
\hline \multirow[t]{2}{*}{ Ensemble Models } & \multicolumn{2}{|l|}{$\mathbf{B A}$} & \multicolumn{2}{|l|}{$\mathbf{A U C}$} & \multicolumn{2}{|l|}{ MAE } & \multicolumn{2}{|l|}{ Time } \\
\hline & UFN & BET & UFN & BET & UFN & BET & UFN & BET \\
\hline Ensemble Selection & 77.8 & 83.3 & 85.9 & 91.0 & 32.2 & 24.0 & 40.13 & 12.01 \\
\hline Cascade Generalization & 80.8 & 83.1 & 86.8 & 90.5 & 27.0 & 22.0 & 72.89 & 40.43 \\
\hline Voting & 83.2 & 81.1 & 77.3 & 76.2 & 16.7 & 18.41 & 11.52 & 3.12 \\
\hline Grading & 77.4 & 79.5 & 77.4 & 79.8 & 22.65 & 20.2 & 195.59 & 51.11 \\
\hline \multirow[t]{2}{*}{ Stacking } & 81.4 & 80.9 & 80.8 & 87.3 & 30.1 & 25.1 & 125.82 & 36.8 \\
\hline & \multicolumn{8}{|c|}{ BoW } \\
\hline Ensemble Selection & 78.9 & 66.1 & 87.9 & 74.0 & 31.7 & 40.4 & 77.65 & 17.75 \\
\hline Cascade Generalization & 86.1 & 56.1 & 92.0 & 62.2 & 21.94 & 44.3 & 94.39 & 37.3 \\
\hline Voting & 86.7 & 62.4 & 85.8 & 62.2 & 13.3 & 37.3 & 14.87 & 6.14 \\
\hline Grading & 83.4 & 59.1 & 83.4 & 59.1 & 16.5 & 39.9 & 346.02 & 64.86 \\
\hline \multirow[t]{2}{*}{ Stacking } & 86.6 & 55.9 & 85.8 & 63.9 & 23.6 & 56.3 & 205.3 & 50.3 \\
\hline & \multicolumn{8}{|c|}{ IG } \\
\hline Ensemble Selection & 80.6 & 66.7 & 88.4 & 74.9 & 31.0 & 40.1 & 47.18 & 32.88 \\
\hline Cascade Generalization & 87.2 & 61.0 & 92.7 & 67.9 & 21 & 43.3 & 146.19 & 108.55 \\
\hline Voting & 89.3 & 64.2 & 84.1 & 61.9 & 10.67 & 35.4 & 24.63 & 18.38 \\
\hline Grading & 85.8 & 62.2 & 85.8 & 60.8 & 14.1 & 39.3 & 262.4 & 101.1 \\
\hline Stacking & 87.1 & 54.5 & 92.5 & 60.6 & 19.47 & 46.9 & 232.62 & 61.05 \\
\hline
\end{tabular}




\section{Table 7 (on next page)}

Summary of the overall results obtained from the best ML model, EL model, and features 
1 Table 7:

2 Summary of the overall results obtained from the best ML model, EL model, and features

\begin{tabular}{|l|l|l|l|l|l|l|}
\hline & Classifier & UFN & Feature & Classifier & BET & Feature \\
\hline \multirow{3}{*}{ Time } & SVM & 0.15 & BoW & SVM & 0.17 & IG \\
\cline { 2 - 8 } & Voting & 11.52 & Tri-gram & Voting & 3.12 & Tri-gram \\
\hline \multirow{2}{*}{ MAE } & SVM & 12.2 & IG & SVM & 23.5 & Tri-gram \\
\cline { 2 - 8 } & Voting & $\mathbf{1 0 . 6 7}$ & IG & Voting & 18.41 & Tri-gram \\
\hline \multirow{2}{*}{ AUC } & SVM & 87.3 & IG & SVM & 76.3 & Tri-gram \\
\cline { 2 - 8 } & Cascade Learning & 92.7 & IG & Ensemble Learning & 91.0 & Tri-gram \\
\hline \multirow{2}{*}{ BA } & SVM & 87.3 & IG & SVM & 76.3 & Tri-gram \\
\cline { 2 - 8 } & Voting & 89.3 & IG & Voting & 83.3 & Tri-gram \\
\hline
\end{tabular}

3 